\title{
Multiple P-wave Morphologies in Paroxysmal Atrial Fibrillation Patients During Sinus Rhythm: A Simulation Study
}

\author{
Dimitris Filos $^{1}$, Paschalis Korosoglou ${ }^{2}$, Dimitris Tachmatzidis ${ }^{3}$, Nicos Maglaveras ${ }^{1}$, Vassilios \\ Vassilikos $^{3}$, Ioanna Chouvarda ${ }^{1}$ \\ ${ }^{1}$ Lab of Computing, Medical Informatics and Biomedical Imaging Technologies, School of Medicine, \\ Aristotle University of Thessaloniki, Thessaloniki, Greece \\ ${ }^{2}$ IT Center, Aristotle University of Thessaloniki, Thessaloniki, Greece \\ ${ }^{3} 3$ rd Cardiology Department, Aristotle University of Thessaloniki, Thessaloniki, Greece
}

\begin{abstract}
$P$-wave in electrocardiographic (ECG) signals represents the activation of the atrial substrate. Its shape is influenced by the origin of the sinus rhythm (SR) and the conduction of each part of the atria and thus the analysis of its morphology $(P W M)$ can reveal information related to the atrial conduction routes. Recently, the analysis of the PWM from PAF patients during SR, revealed the presence of a distinct secondary morphology in a remarkable percentage of beats. We hypothesize that the variability on the PWM can be attributed to the coexistence of electrical remodeling and the transient modification of the conduction route which is the result of the shift of the stimulation site within the sinus node. In this study, the Courtemanche et al. model was incorporated in a $2 D$ tissue of the left and the right atrium, while tissue heterogeneities were considered. Simulations were conducted, using the CHASTE platform, in normal and electrical remodeled tissue. In each simulation, the stimulation site was shifted within the sinus node. The results show that in electrical remodeled tissue, the shift of the stimulation site led to significant changes in the activation time in the atria compared with the healthy tissue. In future, the use of a $3 D$ model of human atria is necessary in order to investigate the reproducibility of the results while the conditions under which the pacemaker location shifts must be also revealed.
\end{abstract}

\section{Introduction}

Atrial fibrillation (AF) is the most common sustained cardiac arrhythmia and it is associated with increased morbidity and mortality. AF is characterized by an abnormal rapid activation of the atria resulting in the reduction of contractility in the atrial muscles. AF is a progressive disease which causes electrophysiological changes in the atrial myocardium [1]. In patients with paroxysmal AF (PAF), electrical remodelling is observed, while in more persistent conditions structural remodelling is also present [2].

More macroscopically, multiple studies revealed that the analysis of the P-wave characteristics in the surface electrocardiographic (ECG) recordings, during SR, can reveal information related to the atrial substrate [3]. According to [4], P-wave morphology depends on (1) the origin of the SR as well as (2) the conduction route. In addition, the variability of the P-wave morphology can be attributed to the transient switch from one group of pacemaking cell to another, within the SA node, or on the different activation routes [5]. The existence of a distinct secondary P-wave morphology in PAF patients was found in [6], while the percentage of beats matching the main or the secondary P-wave morphology can be a predictive metric for the success of PV isolation [7] or for the prediction of AF initiation [8].

In order to understand the underline mechanisms of these phenomenological observations related to arrhythmogenesis, computational modeling constitutes an emerging area of research [9]. Computer simulations have the main advantage that repeated experiments can be performed in a controlled environment while they can shed light on the phenomenological organ level findings such as those detected in electrocardiographic (ECG) recordings and the multiscale mechanisms. In this work, the hypothesis under examination is that the variable location of the pacemaker origin combined with the electrical remodeling, observed in PAF patients, can facilitate the modification of conduction routes leading to the multiple $\mathrm{P}$-wave morphologies. Towards this goal, a simplified 2D atrial geometry, from both atria, was created and the activation of the atria was observed, when the stimulus was originated from different parts within the SA node.

\section{Methodology}

In this study, both atria were studied using $2 \mathrm{D}$ tissue of 
$5 \times 10 \mathrm{~cm}$. The geometric representation of the atria is depicted in Fig1. A number of Pectinate Muscles (PM) were included along the Crista terminalis (CT) as parallel ridges that run on the Right Atrium (RA). The width of the CT were set equal $0.6 \mathrm{~cm}$ while for the PM the width was found to be close to $0.2 \mathrm{~cm}$ [10]. The stimulation originated from an area of $1 \mathrm{~cm}^{2}$ representing the SAN area [11]. Regarding the Left atrium (LA), 2 PVs were included in the model which cover an area of $1 \mathrm{~cm}^{2}$ [12] and were considered as 0 conductivity. The electrical activation of the LA was considered to happen through the CT.

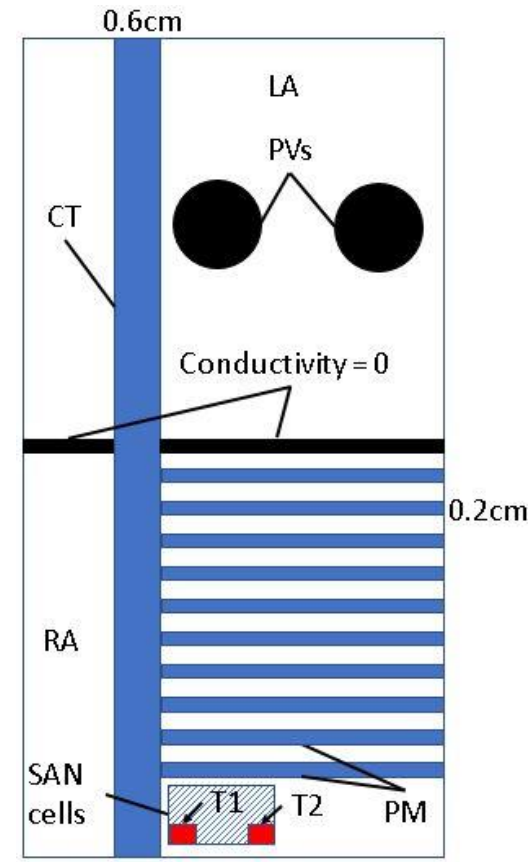

Figure 1 Tissue model of both atria (RA: Right Atrium, LA: Left Atrium, CT: Crista Terminalis, PM: Pectinate muscles, PV: Pulmonary Veins). T1 and T2 represents the two stimulation sites considered in this study.

The electrophysiological model proposed by Courtemanche, Ramirez and Nattel (CRN) [13] was used in the current study in order to describe the electrical activity of the atria. Based on [10] the conductance of three ion currents $\mathrm{I}_{\mathrm{t}}, \mathrm{I}_{\mathrm{CaL}}$ and $\mathrm{I}_{\mathrm{Kr}}$ was adjusted in the different regions of the RA atria (Table 1). Based on the simulations, the cells in the CT region present a prolonged Action Potential Duration (APD) and an enlarged plateau voltage compared to the physiological atrial tissue. Regarding the PM the APD was similar as the atrial tissue.

Apart from the differences in the conduction values of the different atrial structures, anisotropy was also considered. In this respect, monodomain model of electrical current flow was adopted and differences along the longitudinal and transverse were applied. The ratio of the conductivity along the transversal and longitudinal dimension for the conduction bundles (CT and PM) was set to $1: 9$ with the longitudinal direction being parallel to the main axis of the bundles. For the rest of the atrial tissue, the conduction was considered to be isotropic. The transversal conductivity was set to $0.15 \mathrm{~S} / \mathrm{m}$ for both CT and the atrial tissue whereas this value was set equal to 0.11 $\mathrm{S} / \mathrm{m}$ for PM [10].

Table 1. Ionic properties of different atrial structures (RA and LA is for the left and Right atrium respectively, CT is for the Crista Terminalis and PM for the Pectinate Muscles)

\begin{tabular}{lccc}
\hline Conductance & RA, PM & CT & LA \\
\hline $\mathrm{g}_{\text {to }}$ & 1.0 & 0.5 & 1.0 \\
$\mathrm{~g}_{\mathrm{CaL}}$ & 1.0 & 1.0 & 0.67 \\
$\mathrm{~g}_{\mathrm{Kr}}$ & 1.0 & 0.5 & 1.6 \\
\hline
\end{tabular}

In patients with PAF, electrical remodelling is present. The experimental data of ionic channel conductance changes related to PAF have been incorporated in the CRN model of human atrial action potential (AP). Based on [14] the conductance of IK1 was increased by $100 \%$ in order to reproduce atrial remodelling under PAF conditions. The extent of the atrial region where electrical remodelling is present is also a factor that may affect the analysis. In the current work, the atrial remodelling was uniformly considered only in the RA while the LA presents healthy electrophysiological properties.

The stimulation protocol included 4 consecutive stimuli at the same areas and a fifth originating either from the same areas (T1) or from another within the SAN, far from the first 4 areas (T2). The frequency of the excitation was approximately $60 \mathrm{bpm}$ and the duration of each stimulus was set equal to $2 \mathrm{msec}$.

Chaste (Cancer, Heart and Soft Tissue Environment) was used in order to run the simulations described above. Chaste is a general purpose simulation package aimed at multi-scale, computationally demanding problems arising in biology and physiology [15].

\section{Results}

Under health conditions, as can be observed from Fig.2, when the stimulation site of the SAN areas was close to the CT (T1), the activation reaches CT and PM, spreads through the RA and it passes in the LA faster that the case of T2. According to (Table 1) the activation time of each part of the atria does not reveal significant changes (approximately $5 \mathrm{msec}$ ), suggesting that the conduction is rather synchronised even for a shift of the stimulation site. In this respect the P-waves have the potential to match a main morphology. In more details, in both cases, the depolarization of the RA is completed approximately $70 \mathrm{msec}$ after the application of the stimulus, whereas the difference in time for the completion of the depolarization of the LA is only $5 \mathrm{msec}$ between the two cases. 


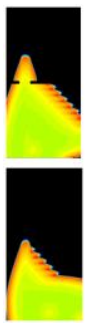

a)

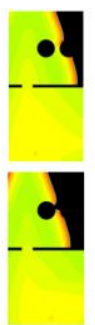

b)

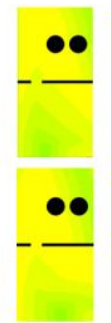

c)

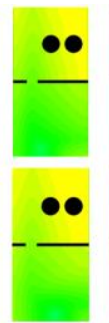

d)

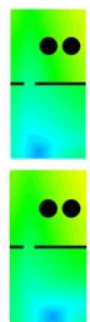

e)

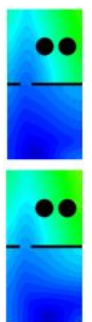

f)

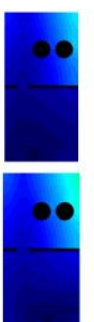

g)

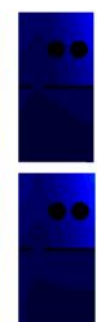

h)
Figure 2 Progression of repolarization in the healthy atria the stimulation originated from the left border of the SAN (top) and form the right border (bottom) at time $50 \mathrm{msec}$ (a), $100 \mathrm{msec}$ (b), $150 \mathrm{msec}$ (c), $200 \mathrm{msec}$ (d), $250 \mathrm{msec}(\mathrm{e}), 300 \mathrm{msec}(\mathrm{f}), 350 \mathrm{msec}(\mathrm{g})$ and $400 \mathrm{msec}(\mathrm{h})$ after the stimulation. Red color denotes greater values while the darkest colors represent values close to $-80 \mathrm{mV}$.

On the other hand, Figure 3 depicts the progression of the transmembrane voltage in an atrial tissue with RA atrial remodelling. It can be observed that the introduction of electrical remodelling in the RA, a realistic assumption of a PAF patient, leads to a faster depolarization and repolarisation of the RA, while the activation time in the LA does not differ with that observed in the healthy subjects. This was expected as the action potential of the electrical remodelled cells have shorter APD. Furthermore, the shift of the stimulation site resulted in significant differences in the depolarization time of the RA which can be correlated to the experimental findings of the existence of a distinct secondary P-wave morphology which appears in patients with PAF [6].

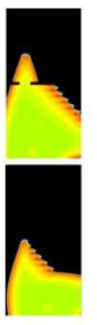

a)

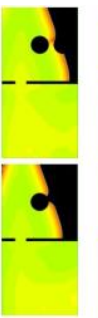

b) c)

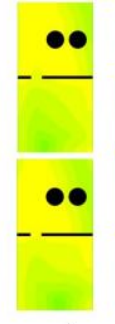

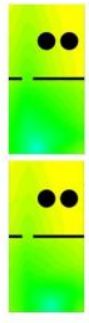

d)

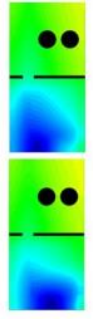

e)

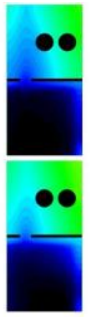

f)

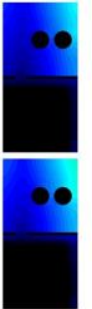

g)

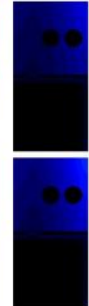

h)
Figure 3 Transmembrane voltage in the atria with RA electrical remodeling. The stimulation originated from the left border of the SAN (top) and form the right border (bottom). The times for a-h, mentioned in the figure, are described in the legend of figure 2 .

In this respect, when the stimulus is close to the CT (T1), the repolarization of the LA starts after $36 \mathrm{msec}$ whereas in the $\mathrm{T} 2$ case after $45 \mathrm{msec}$. In addition, the difference in time for the completion of the RA depolarization is approximately $15 \mathrm{msec}$, while, the related difference for the completion of the LA repolarization is $20 \mathrm{msec}$. These results denote that even in the case of a mild electrical remodelling in the RA, the differences in the activation times are more apparent when a transient shift of the stimulation area occurs. Table 1 provides more details on the different time which was required in order to complete several procedures in the RA and LA.

Table 2. Time spent (in msec) for the completion of each procedure for stimulation applied in $\mathrm{T} 1$ and $\mathrm{T} 2$ under healthy and PAF conditions.

\begin{tabular}{lcccc}
\hline Procedure & \multicolumn{2}{c}{$\begin{array}{c}\text { Healthy } \\
(\mathrm{msec})\end{array}$} & \multicolumn{2}{c}{$\begin{array}{c}\text { PAF } \\
(\mathrm{msec})\end{array}$} \\
\cline { 2 - 5 } & $\mathrm{T} 1$ & $\mathrm{~T} 2$ & $\mathrm{~T} 1$ & $\mathrm{~T} 2$ \\
\hline $\begin{array}{l}\text { Reach fast conduction } \\
\text { routes }\end{array}$ & 9 & 13 & 9 & 13 \\
$\begin{array}{l}\text { LA depolarization start } \\
\begin{array}{l}\text { RA depolarization } \\
\text { completion }\end{array}\end{array}$ & 39 & 45 & 36 & 45 \\
$\begin{array}{l}\text { LA depolarization } \\
\text { completion }\end{array}$ & 68 & 70 & 63 & 76 \\
$\begin{array}{l}\text { RA repolarization start } \\
\begin{array}{l}\text { LA repolarization start } \\
\text { RA repolarization }\end{array}\end{array}$ & 280 & 285 & 235 & 250 \\
completion & 475 & 320 & 283 & 306 \\
\hline
\end{tabular}

\section{Discussion and conclusions}

This work focuses on patterns of electrical activation progression in both atria and presents the initial comparative analysis com results as regards to healthy subjects and those with PAF in order to understand the existence of multiple $\mathrm{P}$-wave morphologies in both categories. According to previous studies, P-wave morphology was found to be influenced by the existence of ectopic foci, but this study is the first to investigate the effect of the modification of the conduction routes, and thus the variations of the P-wave morphologies, when a transient shift of the pacemaker activity, within the SAN is present. The analysis was based on simulations performed in a 2D tissue, including the main anatomical structures of the right and left atrium. The simulations were performed in both healthy and PAF conditions, where electrical remodeling was considered. In this work, the electrical remodeling was considered only in the RA.

According to the initial results, the variation of the activation origination in a region of $1 \mathrm{~cm}^{2}$ resulted in small modifications of the activation pattern and thus it confirms the research findings that multiple $\mathrm{P}$-waves may be found in both healthy and PAF conditions. Furthermore, the modifications of the activation patterns are more apparent, in patients with PAF, whereas in healthy subjects the pacemaking shift does not result on remarkable changes of the activation routes. Since the first part of P-wave corresponds mainly to RA depolarization while the second part to the LA depolarization [16], the differences in the 
activation time presented in the current work may explain the deflection of the P-wave characteristics in a beat to beat basis. In particular, those findings can explain the greater percentage of beats matching a distinct secondary morphology in PAF patients.

\section{Limitations}

Apart from the aforementioned analysis; the factors, like autonomic remodeling [17], which result in the variations of the pacemaking activity, must be studied. In this respect, the effect of the ANS and the Achetylcholine on the frequency of those variations must be considered. In addition, the analysis performed was based on a $2 \mathrm{D}$ tissue which represents a realistic atrial geometry, but not fully detailed with the fiber orientation and all the anatomical structures. Further simulations must be performed to confirm the initial finding presented here. Finally, anatomical models of human torso must also be considered to reconstruct the ECG recording and thus correlate the $\mathrm{P}$ wave morphology deflections with the underground electrical activation of the atria.

\section{Acknowledgements}

Results presented in this work have been produced using the AUTH Compute Infrastructure and Resources. The authors would like to acknowledge the support provided by the Scientific Computing Office throughout the progress of this research work.

\section{References}

[1] M. C. E. F. Wijffels, C. J. H. J. Kirchhof, R. Dorland, and M. A. Allessie, "Atrial Fibrillation Begets Atrial Fibrillation: A study in awake chronically instrumented Goats," Circulation, vol. 92, no. 7, pp. 1954-1968, 1995.

[2] P. Kirchhof, S. Benussi, D. Kotecha, A. Ahlsson, D. Atar, B. Casadei, M. Castella, H.-C. Diener, H. Heidbuchel, J. Hendriks, G. Hindricks, A. S. Manolis, J. Oldgren, B. A. Popescu, U. Schotten, B. van Putte, and P. Vardas, "2016 ESC Guidelines for the management of atrial fibrillation developed in collaboration with EACTS," Eur. Heart J., vol. 37, pp. 2893-2962, 2016.

[3] M. Saha, G. Conte, M. L. Caputo, F. Regoli, R. Krause, A. Auricchio, and V. Jacquemet, "Changes in P-wave morphology after pulmonary vein isolation: insights from computer simulations.," Europace, vol. 18, no. suppl 4, pp. iv23-iv34, Dec. 2016.

[4] P. G. Platonov, "P-wave morphology: Underlying mechanisms and clinical implications," Ann. Noninvasive Electrocardiol., vol. 17, no. 3, pp. 161-169, 2012.

[5] O. Monfredi, H. Dobrzynski, T. Mondal, M. R. Boyett, and G. M. Morris, "The anatomy and physiology of the sinoatrial node-A contemporary review," PACE - Pacing and Clinical Electrophysiology, vol. 33, no. 11. pp. 1392-1406, 2010.

[6] D. Filos, I. Chouvarda, D. Tachmatzidis, V. Vassilikos, and N. Maglaveras, "Beat-to-beat P-wave morphology as a predictor of paroxysmal atrial fibrillation," Comput. Methods Programs Biomed., vol. 151, pp. 111-121, 2017.

[7] Y. Huo, F. Holmqvist, J. Carlson, T. Gaspar, G. Hindricks, C. Piorkowski, A. Bollmann, and P. G. Platonov, "Variability of P-wave morphology predicts the outcome of circumferential pulmonary vein isolation in patients with recurrent atrial fibrillation," J. Electrocardiol., vol. 48, no. 2, pp. 218-225, 2015.

[8] A. Martínez, R. Alcaraz, and J. J. Rieta, "Study on the Pwave feature time course as early predictors of paroxysmal atrial fibrillation.," Physiol. Meas., vol. 33, no. 12, pp. 195974, 2012.

[9] D. M. Bers and E. Grandi, "Human Atrial Fibrillation: Insights from computational electrophysiological models," Trends Cardiovasc. Med., vol. 21, no. 5, pp. 145-150, 2011.

[10] A. Ferrer, R. Sebastián, D. Sánchez-Quintana, J. F. Rodríguez, E. J. Godoy, L. Martínez, and J. Saiz, "Detailed anatomical and electrophysiological models of human atria and torso for the simulation of atrial activation," PLoS One, vol. 10, no. 11, p. e0141573, 2015.

[11] S. Y. Ho, R. H. Anderson, and D. Sánchez-Quintana, "Atrial structure and fibres: Morphologic bases of atrial conduction," Cardiovasc. Res., vol. 54, no. 2, pp. 325-336, 2002.

[12] N. Virag, V. Jacquemet, C. S. Henriquez, S. Zozor, O. Blanc, J.-M. Vesin, E. Pruvot, and L. Kappenberger, "Study of atrial arrhythmias in a computer model based on magnetic resonance images of human atria.," Chaos, vol. 12, no. 3, pp. 754-763, Sep. 2002.

[13] M. Courtemanche, R. J. Ramirez, and S. Nattel, "Ionic targets for drug therapy and atrial fibrillation-induced electrical remodeling: Insights from a mathematical model," Cardiovasc. Res., vol. 42, no. 2, pp. 477-489, 1999.

[14] C. Tobón, M. Duarte, J. E. Duque, M. A. Becerra, S. S. Arango, K. Cardona, and J. Saiz, "Chloroquine effect on rotor termination under paroxysmal and chronic atrial fibrillation. 2D simulation study," in Proceedings of the 2014 IEEE Central America and Panama Convention, CONCAPAN 2014, 2014.

[15] G. R. Mirams, C. J. Arthurs, M. O. Bernabeu, R. Bordas, J. Cooper, A. Corrias, Y. Davit, S. Dunn, A. G. Fletcher, D. G. Harvey, M. E. Marsh, J. M. Osborne, P. Pathmanathan, J. Pitt-francis, J. Southern, N. Zemzemi, and D. J. Gavaghan, "Chaste: An Open Source C ++ Library for Computational Physiology and Biology," vol. 9, no. 3, 2013.

[16] A. Loewe, M. W. Krueger, P. G. Platonov, F. Holmqvist, O. Dösse, and G. Seemann, Left and Right Atrial Contribution to the P-wave in Realistic Computational Models, Springer I., vol. 9126 of th. 2015.

[17] J. Pellman and F. Sheikh, "Atrial fibrillation: Mechanisms, therapeutics, and future directions," Compr. Physiol., vol. 5, no. 2, pp. 649-665, Apr. 2015.

Address for correspondence.

Dimitris Filos

Lab of Computing, Medical Informatics and Biomedical Imaging Technologies, Medical school, Aristotle University of Thessaloniki, University campus, Box 323, GR54124, Thessaloniki, Greece dimfilos@auth.gr. 